\title{
A Possible Role for Protein Synthesis, Extracellular Signal-Regulated Kinase, and Brain-Derived Neurotrophic Factor in Long-Term Spatial Memory Retention in the Water Maze
}

\author{
Anne-Marie T. McGauran \\ National University of Ireland Maynooth and Centre de \\ Recherches sur la Cognition Animale \\ Daniel Barry \\ National University of Ireland Maynooth
}

\author{
J. Bernadette Moore and Declan Madsen \\ Institute of Immunology, National University of \\ Ireland Maynooth
}

\author{
Shirley O'Dea and Bernard P. Mahon \\ Institute of Immunology, National University of \\ Ireland Maynooth
}

\author{
Sean Commins \\ National University of Ireland Maynooth
}

\begin{abstract}
Hippocampal protein synthesis is dependent upon a number of different molecular and cellular mechanisms that act together to make previously labile memories more stable and resistant to disruption. Both brain-derived neurotrophic factor (BDNF) and extracellular signal-Regulated kinase (ERK) are known to play an important role in protein synthesis-dependent memory consolidation, via the mitogen-activated protein-kinase (MAP-K) signaling pathway during the transcription phase of protein synthesis. The current study investigates the influence of protein synthesis inhibition (PSI) by cycloheximide on spatial learning and memory. In an initial experiment, the authors utilized two doses of cycloheximide $(0.5$ $\mathrm{mg} / \mathrm{kg}$ and $1.0 \mathrm{mg} / \mathrm{kg}$, intraperitoneally) to determine the dose at which long-term ( $>24$ hours) memories are impaired. A second experiment was designed to investigate the effect of PSI on the formation of cue-platform associations in the watermaze, and on BDNF and ERK expression in the hippocampus. At the higher dose $(1.0 \mathrm{mg} / \mathrm{kg})$ cycloheximide resulted in impaired retention of the water maze. BDNF and ERK expression was also down-regulated in animals injected with this dose of cycloheximide. Our results demonstrate a role of protein synthesis in spatial memory retention, along with a possible relationship between protein synthesis and hippocampal BDNF/ERK expression.
\end{abstract}

Keywords: memory consolidation, protein synthesis, BDNF, ERK, hippocampus

Consolidation of long-term memory is thought to occur via molecular and cellular processes, which over time allow memories to be strengthened and become resistant to disruption (McGaugh, 1999, 2000; Müller \& Pilzecker, 1900). Memory consolidation involves neural processes occurring at both cellular and systems levels. During consolidation, new proteins are synthesized to allow

Anne-Marie T. McGauran, Department of Psychology, National University of Ireland Maynooth, Co. Kildare, Ireland and Centre de Recherches sur la Cognition Animale, CNRS 5169, Université Paul Sabatier, Toulouse, France; J. Bernadette Moore, Declan Madsen, Shirley O'Dea, and Bernard P. Mahon, Institute of Immunology, National University of Ireland Maynooth, Co. Kildare, Ireland; Daniel Barry and Sean Commins, Department of Psychology, National University of Ireland Maynooth, Co. Kildare, Ireland.

This work was supported by the Irish Research Council for the Humanities and Social Sciences, EUFP6 Marie Curie Actions and the Department of Psychology, NUI Maynooth. We thank Dr Pascal Roullet (CNRSToulouse) for his suggestions and contributions.

Correspondence concerning this article should be addressed to Sean Commins, Department Psychology, National University of Ireland Maynooth, Maynooth, CO Kildare, Ireland. E-mail: sean.commins@nuim synaptic strengthening, and cellular consolidation occurs when a memory changes from being protein synthesis dependent to independent. System consolidation, on the other hand, refers to the process whereby a memory becomes independent of the hippocampus and distributed through the neocortex (see Dash et al., 2004, for a review).

Investigations into the role of protein synthesis in memory consolidation have reported impairing effects due to protein synthesis inhibition (Agnihotri, Hawkins, Kandel, \& Kentros, 2004; Dudai \& Eisenburg, 2004). Injections of the protein synthesis inhibitor anisomycin into the amygdala and the hippocampus, for example, impair consolidation for contextual fear-conditioning tasks (Debiec, LeDoux, \& Nader, 2002; Fischer, Sananbenesi, Schrick, Spiess, \& Radulovic, 2004), while microinjections of anisomycin into the lateral ventricle inhibits long-term consolidation of spatial learning in the water maze task (Meiri \& Rosenblum, 1998). One mechanism by which protein synthesis can be triggered is via the mitogen-activated protein-kinase (MAP-K) signaling cascade. This pathway is responsible for the production of extracellular signal-regulated kinase (ERK; Kaplan \& Miller, 2000; Segal \& Greenberg, 1996), which in turn plays a major role 
in protein synthesis (Adams \& Sweatt, 2002). As such, the MAP-K signaling cascade, and ERK in particular, has been frequently demonstrated as playing an important role in learning and memory. This role has been seen in numerous different behavioral paradigms, each testing different aspects of memory formation, such as the watermaze (Atkins, Selcher, Petraitis, Trzaskos, \& Sweatt, 1989; Blum, Moore, Adams, \& Dash, 1999; Selcher, Atkins, Trzaskos, Paylor, \& Sweatt, 1999), conditioned taste aversion (Bermen et al., 1998), and long-term potentiation (LTP) studies (English \& Sweatt, 1997).

Another molecule that plays a critical role in learning and memory is brain-derived neurotrophic factor (BDNF). Along with its neurotrophin-related role in regulating the survival and regeneration of neurons (Davies, 1994; Lewin \& Barde, 1996), BDNF has been shown to play a vital role in learning and memory in mice (Bao, Chen, Qiao, Knusel, \& Thompson, 1998), monkeys (Tokuyama, Okuno, Hashimoto, Li, \& Miyashita, 2000), and chicks (Johnston, Clements, \& Rose, 1999) (see Yamada, Mizuno, \& Nabeshima, 2002, for a review). With the highest expression of BDNF in the brain found in the hippocampus, BDNF has also been strongly implicated in hippocampally dependent spatial learning (Hall, Thomas, \& Everitt, 2000; Kesslak, So, Choi, Cotman, \& Gomez-Pinilla, 1998; Yamada et al., 2002). In situ hybridization techniques have revealed a rapid induction of BDNF in the CA1 subfield after hippocampally dependent contextual learning (Hall et al., 2000), while watermaze training results in increased expression of BDNF in the hippocampus, but not in the cerebellum, striatum or neocortex (Kesslak et al., 1998).

By phosphorylating the TrkB receptor, BDNF can directly influence the onset of a number of signaling pathways (Derkinderen, Enslen, \& Girault, 1999) including the MAP-K signaling cascade. Specifically it is thought that BDNF/TrkB signaling converges on the MAP-K pathway to increase excitatory synaptic transmission in hippocampal-dependent learning tasks (Tyler, Alonso, Bramham, \& Pozzo-Miller, 2002). As both ERK and BDNF act through the MAP-K signaling cascade during the transcription phase of protein synthesis (Adams \& Sweatt, 2002; Derkinderen et al., 1999), interactions between ERK and BDNF in protein-synthesis dependent spatial learning may play an intrinsic role in the formation of long-term spatial memories. There is some evidence to suggest that this may be the case. Inhibition of ERK, for example, results in down-regulated BDNF activity (Han \& Holtzmann, 2000), while spatial learning has been found to lead to an upregulation of both ERK and BDNF expression in the dentate gyrus (Gooney et al., 2001).

We have demonstrated previously that retention of spatial memories in the water maze task relies on the learned association between the location of the hidden platform and environmental visual cues. By rotating the cues $180^{\circ}$ seven days post acquisition we found that animals rotated their searching strategy by a similar amount (McGauran, Harvey, Cunningham, Craig, \& Commins, 2004). We hypothesize that this specific learned and consolidated association between the platform and the environmental cues may be reliant on the synthesis of hippocampal proteins.

As consolidation of retained information is usually tested by administration of an intervention immediately (or at some critical period) postacquisition (Bourtchouladze et al.., 1998; Grecksch \& Matthies, 1980; Quevedo et al., 1999; Rossato et al., 2007), it is often difficult to assess when learning may occur in the typically adopted spaced-training version of the watermaze task. We, for example, normally train animals for 4 trials per day for 5 days (see Commins, Cunningham, Harvey, \& Walsh, 2003, and also D’Hooge \& De Deyn, 2001; Morris, 1984). As this task involves multiple training trials over many days, consolidation, or indeed reconsolidation, may occur repeatedly over the training period. By administering the protein synthesis inhibitor (PSI) cycloheximide (CHX) immediately posttraining on each of the 5 acquisition days we aim, in the first experiment, to determine the general effectiveness of this inhibitor on general performance in the water maze task. We would not envisage $\mathrm{CHX}$ to have a within-day effect (affecting performance from Trial 1-Trial 4 see Meiri \& Rosenblum, 1998) but would however expect to find a 24-hr performance effect (Bear et al., 1995). To test for this, we will analyze the performance of each treated group on the first trial on each of Days 2, 3, 4, and 5 of training. In addition, continuing our previous investigations into memory retention involving longer intervals (7 days, e.g., McGauran et al., 2004, McGauran, O’Mara, \& Commins, 2005) we specifically wish to determine the longer-term effectiveness of this PSI ( $>24$ hours) on the retention of spatial memories.

In order for memory consolidation to be completely blocked, it is thought that protein synthesis must be inhibited by at least $90 \%$ (Meiri \& Rosenblum, 1998), though this may depend on the intensity of the training provided, where an increase in the intensity of training is thought to decreases the effectiveness of protein inhibition. Very little research, however, has been performed into the dose-dependent nature of CHX, with studies reporting impairing effects with both low doses (e.g., Luft, Buitrago, Kaelin-Lang, Dichgans, \& Schulz, 2004) and much higher doses (Hirate et al., 1997) of the drug. A single CHX dose of $0.6 \mathrm{mg} / \mathrm{kg}$, for example, blocks protein synthesis by only $75 \%$ (e.g., Lu, Lin, \& Peng, 2004; Luft et al., 2004). By using two dose levels in the current study $(0.5 \mathrm{mg} / \mathrm{kg}$ and $1.0 \mathrm{mg} / \mathrm{kg})$ we wish to determine the best dose at which long-term memories are impaired. It is anticipated that, based on this previous research, performance should not be completely impaired after $0.5 \mathrm{mg} / \mathrm{kg}$, while, on the other hand, at 1.0 $\mathrm{mg} / \mathrm{kg}$, protein synthesis should be inhibited to a sufficient extent to show a significant impairment in memory. This approach allows us to interpret our findings with those of previous research, along with providing a control with which to compare our results.

Having determined both the effectiveness and the appropriate dosage of $\mathrm{CHX}$, we, in a second experiment, will specifically examine the role of protein synthesis on the formation of cueplatform relations. We predict that if these cue-platform relations are dependent upon protein synthesis, then administration of $\mathrm{CHX}$ will impair the animals' ability to follow rotated visual cues while locating the platform. In addition, we will examine the expression of both hippocampal BDNF and ERK in relation to protein synthesis activity and hypothesize that these two molecules will be directly down-regulated by the inhibition of protein synthesis.

\section{Experiment 1. Determining the Effectiveness of CHX in Blocking Spatial Learning}

\section{Materials and Method}

Animals. Male Wistar rats (250-350 g; Biomedical Facility, University College, Dublin) aged approximately 3 months were 
used as subjects. Rats were housed 3 per cage and were kept in a temperature-controlled room that was maintained on a fixed lightdark cycle (7 a.m.-7 p.m.). All rats were given free access to food and drink and were well handled before experimentation. Testing was carried out during the light phase.

Experimental apparatus. A standard water maze procedure was used. This was a black circular pool $(1.7 \mathrm{~m}$ diameter; $35 \mathrm{~cm}$ deep) filled to $31 \mathrm{~cm}$. Rats could escape the water by climbing onto a platform $(29 \mathrm{~cm} \times 9 \mathrm{~cm})$ located in the middle of the northeastern (NE) quadrant of the pool, and submerged $2 \mathrm{~cm}$ below water surface so that it was invisible at water level. Black curtains surrounded the entire maze at a distance of $50 \mathrm{~cm}$ from the pool wall. Distal visual cues included two $(60 \mathrm{~W})$ lights suspended from the ceiling (on the inside of the curtain) at a distance of $75 \mathrm{~cm}$ from the pool wall and at angles of approximately $60^{\circ}$. A rectangular sheet of white paper $(55 \mathrm{~cm} \times 81 \mathrm{~cm})$ attached to the curtain on the eastern side of the pool was also used as a distal cue. One light bulb was suspended from the northwestern (NW) corner, and the other from the NE position. Escape latencies were recorded during each trial using a computerized digital tracking system (EthoVision: Noldus Information Technology, Wageningen, Netherlands).

Behavioral procedure. All animals received identical training of 4 trials per day for 5 days. Animals were carried to and from the maze in a plastic carry box $(30 \times 40 \times 30 \mathrm{~cm})$. They were placed into the maze at starting points in the Northern, Southern, Eastern and Western quadrants in a pseudorandom fashion, and allowed to search for the hidden platform for 60 seconds. If after this time they did not correctly locate the platform, they were led to it by the experimenter. Once on the platform, the animals were allowed to remain there for 15 seconds. Following a further 15 second intertrial-interval animals were then returned to the maze at a new starting point until a total of four trials were completed.

Animals were randomly placed into one of 3 groups $(n=$ 7/group) following the final trial on the first day. Animals in Group 1 (SAL) were administered intraperitoneally (i.p.) with saline $(0.9 \% \mathrm{NaCl}, 1.0 \mathrm{ml} / \mathrm{kg})$, Group 2 (CHX1) were administered i.p. with $0.5 \mathrm{mg} / \mathrm{kg}$ of cycloheximide (in saline, $1.0 \mathrm{ml} / \mathrm{kg}$ ) and Group 3 (CHX2) injected i.p. with $1.0 \mathrm{mg} / \mathrm{kg}$ of cycloheximide (in saline, $1.0 \mathrm{ml} / \mathrm{kg}$ ). All drugs were obtained from Sigma (Ireland). This procedure was repeated following the final trial for each subsequent training day.

Animals were then rested for a week before retention was tested. No drug treatment was given during this period. Retention was assessed 7 days postacquisition via a probe trial, in which the escape platform was removed. Animals were placed into the maze at the NW position and allowed to swim in the water for 60 seconds while their swimming patterns were monitored. The experimental environment of the retention phase remained the same as during the acquisition phase. Measures of retention included the analysis of percentage time spent by each group in the platform area (defined by a circular area centered at the platform in the NE quadrant, with a radius of approximately $27 \mathrm{~cm}$, the body length of a rat) during the initial 30 seconds of the retention phase. This was compared to the time spent in three other equivalent areas in each of the other quadrants (NW, SE, SW). The first 30 seconds were chosen, as we have previously shown that retention is sensitive to disruption after this time period (see McGauran et al., 2004). In addition, the percentage time spent by animals at the side of the pool in an outer corridor area (approximately $20 \mathrm{~cm}$ in width, inside the pool wall) was compared. This area was chosen for analysis as animals demonstrating general memory impairments would often display thigmotactic behavior and tend to remain at the pool edge (Barnett, 1963).

Statistics. A series of repeated and/or mixed analyses of variance (ANOVAs) were used to analyze both acquisition and retention phases. Where appropriate independent and/or dependent $t$ tests were carried out using SPSS (Version 12) $\left({ }^{*} p<.05 ;{ }^{* *} p<\right.$ $\left..01 ;{ }^{* * *} p<.001\right)$.

\section{Results}

Protein synthesis inhibition does not impair acquisition of the watermaze task. All animals from all three groups successfully acquired the water maze task. A $3 \times 5$ mixed-factorial ANOVA found that escape latencies decreased significantly from Day 1 to Day 5 for all groups, $F(4,42)=25.564, p<.001$; Figure 1a. No significant treatment effect was found, $F(2,18)=1.607, p>.05$, and no interaction was found between days and treatment, $F(8$, 72) $=0.711, p>.05$.

As the training protocol took place over a period of 5 days, it was investigated whether posttraining drug administration had an effect on performance in the first trial of the Session 24 hours later, that is, the first trial on Day 2 and the first trial on each of the subsequent training days (3, 4 and 5). Figure $1 \mathrm{~b}$ demonstrates that the mean escape latency for each groups' first trial decreases from Day 2 to Day 5 . A $3 \times 4$ mixed factorial ANOVA confirmed a significant effect for Day, $F(3,57)=24.61, p<.001$, but importantly neither a between groups nor an interaction effect was found, $F(2,19)=1.206, p>.05 ; F(6,57)=0.762, p>.05$, respectively. As escape latencies alone might not reflect spatial learning, we also examined the mean percentage time spent by each group exploring the NE quadrant on the first trial of each day (Figure 1c). Again a mixed factorial ANOVA was conducted and found a significant effect for Day, $F(3,57)=5.782, p<.01]$, suggesting that all groups spent more time in this quadrant with acquisition. We found a small effect for Group, $F=3.3(2,19)$, $2,19, p=.05$, with post hoc tests revealing that the Saline group spent significantly more time searching in the NE quadrant compared to the $\mathrm{CHX} 2$ group (Tukey, $p=.05$ ). In addition, we did not find an interaction effect, $F(6,57)=0.611, p>.05)$.

Protein synthesis inhibition impairs long-term spatial memory retention. In order to establish if $\mathrm{CHX}$ at $0.5 \mathrm{mg} / \mathrm{kg}$ or $1.0 \mathrm{mg} / \mathrm{kg}$ impaired long-term retention (7 days postacquisition), the mean percentage time spent by each group in the platform area (NE) and the four equivalent areas in each of the other quadrants (NW, SE, SW) was examined (Figure 2a, inset). A $3 \times 4$ way ANOVA revealed a main effect for area, $F(3,72)=16.552, p<.001$, and a treatment effect, $F(2,72)=3.549, p<.05$. An interaction between treatment and area was also found, $F(6,72)=3.604, p<$ .01 , and was further investigated by post hoc (Tukey) analysis. Here, we found that both SAL and CHX1 spent significantly more time in the NE platform area than the other three equivalent areas, while CHX2 showed no such preference for the NE area. In addition, there was no significant difference between SAL and CHX1 in the time spent in the NE area, while CHX2 spent significantly less time here than either of the other two groups (Figure 2a). 
a
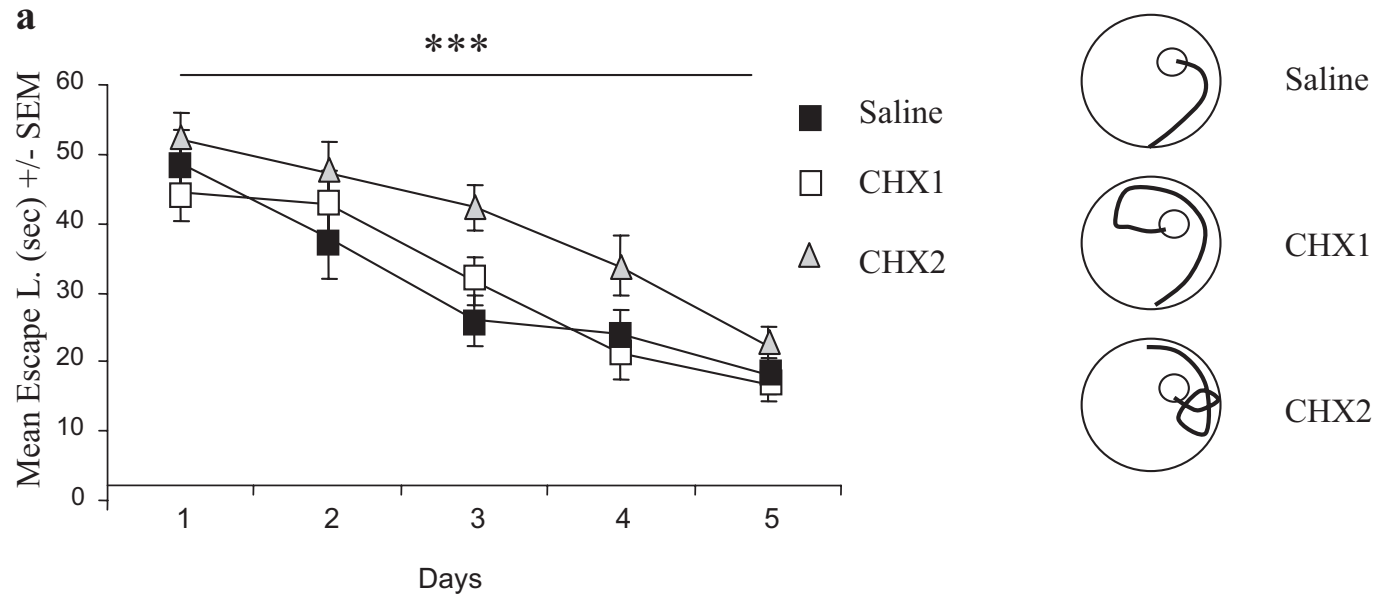

b

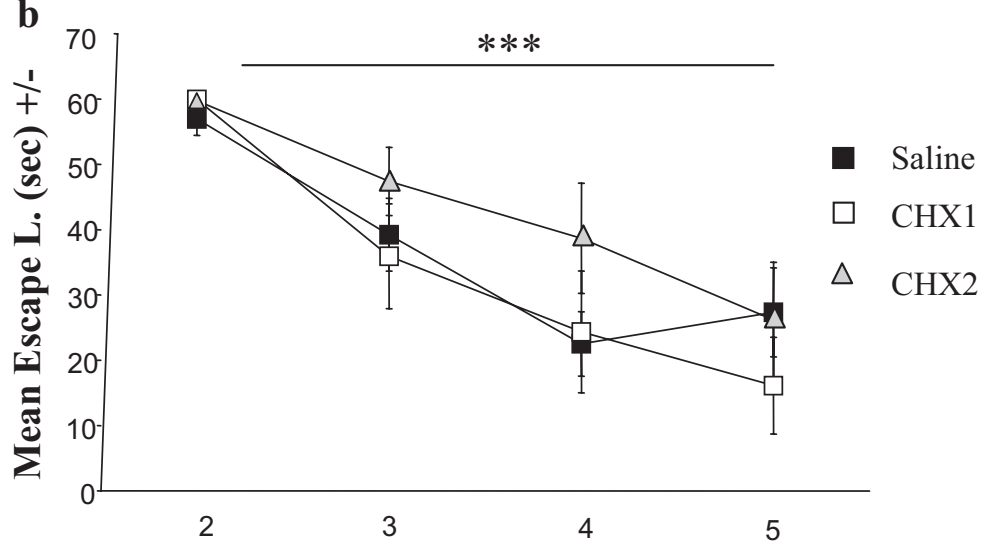

Days (first trial)

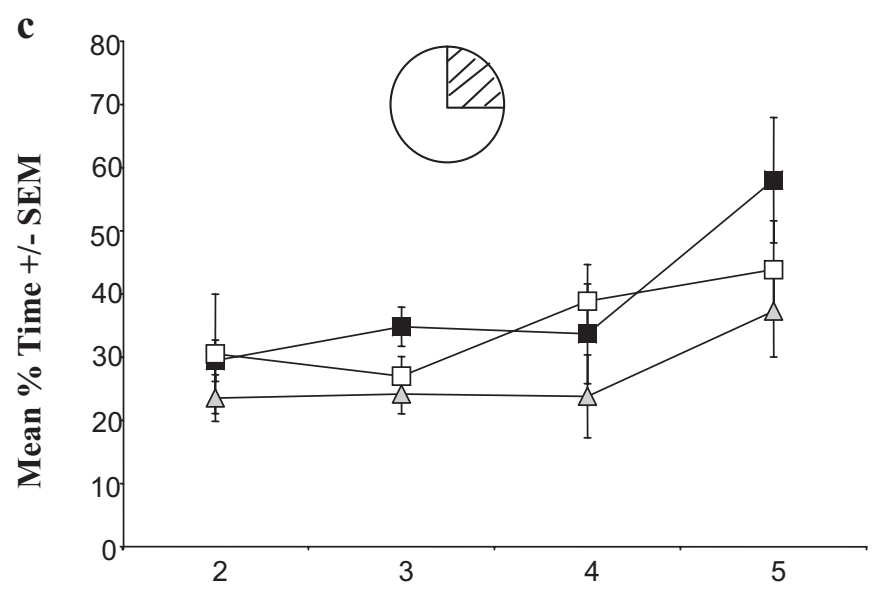

- Saline

$\square \quad \mathrm{CHX} 1$

$\triangle$ CHX2

Days (first trial)

Figure 1. Mean Escape Latencies for all conditions during acquisition phase demonstrating that the overall escape latencies decreased significantly across groups over the 5-day acquisition phase (Experiment 1). Right panel, representative trajectories from each group taken from the final day of acquisition (Day 5). B, A line graph representing the 24-hr influence of each drug on escape latency performance on the first trial of each day. C, A line graph representing the 24-hr influence of each drug by examining the mean percentage time spent by each group in the northeast quadrant (where the hidden platform was located) on the first trial of each acquisition day (subsequent to injection). 

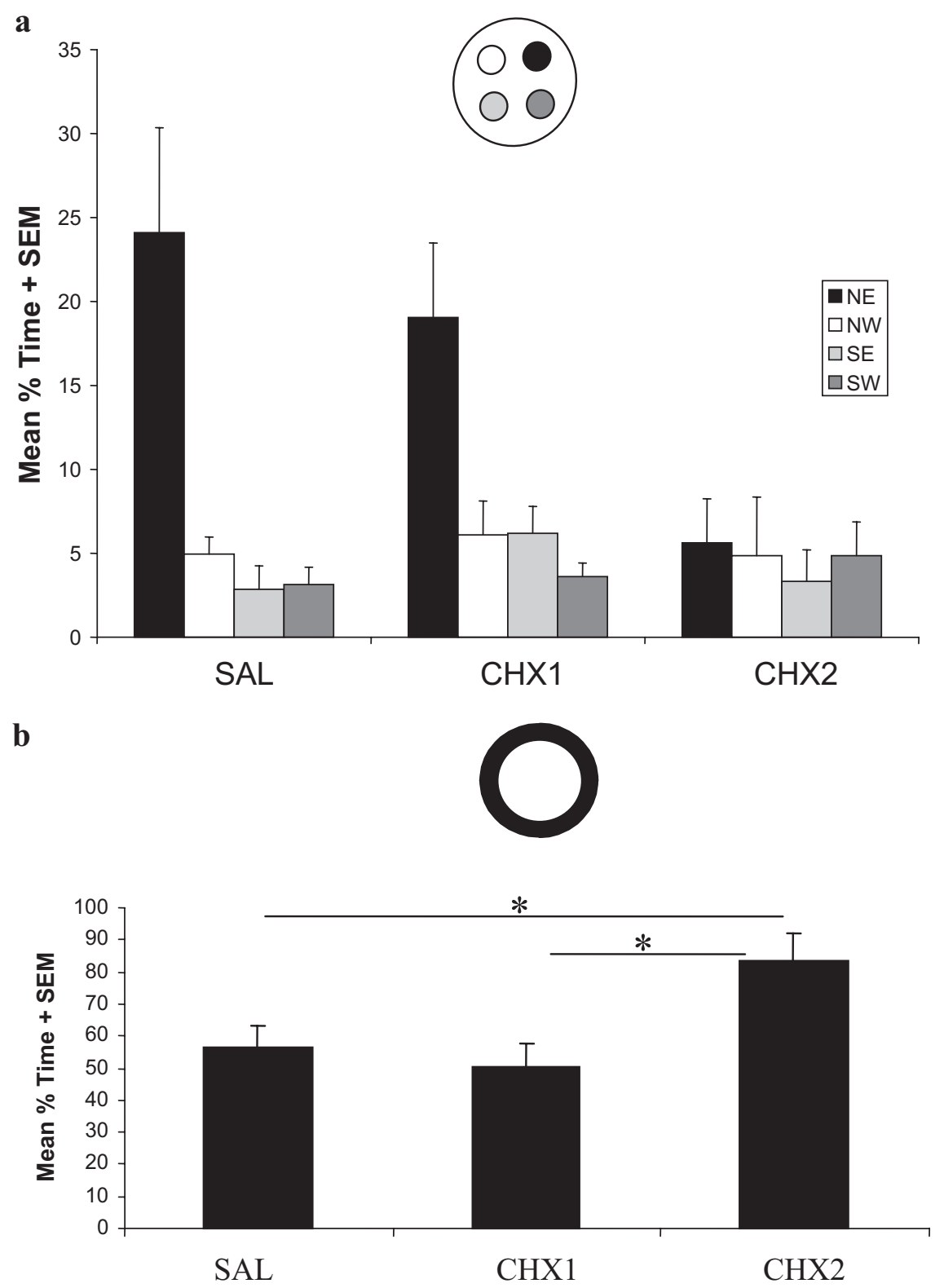

\begin{abstract}
Figure 2. A, Mean percentage time (of the first 30 seconds) of retention spent in the platform and equivalent areas by all groups (see inset). Animals in Group 1 (saline; SAL) and Group 2 (CHX1) both presented a preference for the northeast (NE) platform area over the others, while CHX2 showed no such preference. CHX2 also swam in the NE area significantly less than both SAL and CHX1 groups, demonstrating a dose-dependent effect. B, Mean percentage time (of the first 30 seconds) of retention spent in the outer corridor by all groups. CHX2 swam in this region significantly more than both the SAL group and CHX1, demonstrating again a dose-dependent effect.
\end{abstract}

As a second measurement of impairment, the amount of time spent swimming at the side of the pool was also measured (see Figure $2 \mathrm{~b}$, inset). This has been previously suggested as an indicator of general impairment and confusion in animals navigating the watermaze (McGauran et al., 2005). A significant main effect was found, $F(2,18)=5.979, p=.01$, and post hoc (Tukey) tests revealed that the CHX2 group spent more time swimming in this region than both SAL and CHX1 (Figure 2b).
Experiment 2: Determining the Effect of Protein Synthesis Inhibition on Retention of Cue-Platform Associations and on Hippocampal BDNF and ERK Expression

The results from Experiment 1 demonstrate that, while protein synthesis inhibition did not influence acquisition, CHX administered at $1.0 \mathrm{mg} / \mathrm{kg}$ successfully impaired long-term ( $>24$ hours) 
retention of the maze. Having established in this manner that $\mathrm{CHX}$ produced a dose-dependent effect, with $0.5 \mathrm{mg} / \mathrm{kg}$ having no effect, but $1.0 \mathrm{mg} / \mathrm{kg}$ successfully impairing spatial retention, we proceeded in a second experiment to use this higher dose in examining the role of protein synthesis specifically on the formation of cue-platform associations in the watermaze and importantly how hippocampal BDNF and ERK may be affected.

\section{Materials and Method}

Behavioral procedure. Animals in Experiment 2 underwent identical training to that used in Experiment 1 (4 trials/day for 5 days). Animals ( $n=26$ ) received 5 days of training with four trials per day. They were initially divided into two equally numbered treatment groups: CHX, which received i.p. injection of CHX (1.0 $\mathrm{mg} / \mathrm{kg}$ ) and SAL, which received i.p. saline $(\mathrm{NaCl} 9 \%)$, immediately after the final session on each training day. Seven days postacquisition, animals were tested for retention via a probe trial. However, to examine the effect of $\mathrm{CHX}$ on cue-platform association, animals in both SAL and CHX were further divided into two subgroups: controls (CT) and cue-rotated (CR). For the CT group, the distal cue layout remained identical to that used during training, while for the $\mathrm{CR}$ group the cues were rotated by $180^{\circ}$. Once the 60 -s probe trial was complete, the animals were removed from the maze and replaced to their home cage.

Biochemical procedure. All rats in both CHX and SAL groups were sacrificed immediately after the retention probe trial and their brains were quickly removed. Hippocampal tissues were placed into $1 \mathrm{ml}$ Krebs solution (containing $2 \mathrm{mM} \mathrm{CaCl}_{2}$ and DMSO [1:10]) and were stored at $-20{ }^{\circ} \mathrm{C}$ for later analysis of BDNF and ERK expression.

ELISA Analysis of BDNF Expression. The expression of BDNF in the hippocampal formation was measured via a BDNF Emax ImmunoAssay System (Promega U.K.). Hippocampal samples were homogenized on ice, in ice-cold Krebs solution 50 times. Protein was assessed and samples diluted with Krebs solution to give equal protein concentrations, and stored at $-20{ }^{\circ} \mathrm{C}$. 96-well plates were coated with $100 \mu \mathrm{l}$ anti-BDNF monoclonal antibody diluted $(1: 1000)$ in carbonate coating buffer $(0.025 \mathrm{M}$ sodiumbicarbonate, $0.025 \mathrm{M}$ sodium carbonate, $\mathrm{pH} 9.7)$. Plates were covered and incubated overnight at $4{ }^{\circ} \mathrm{C}$. On day two, plates were washed once in Tris- $\mathrm{HCl}$ wash buffer (20 mM Tris- $\mathrm{HCl}$ (pH 7.6); $150 \mathrm{mM} \mathrm{NaCl} ; 0.05 \%$ Tween vol/vol) to remove excess antibody. They were then blocked for nonspecific binding for 1 hour at room temperature. Postblocking, plates were washed once before $100 \mu \mathrm{l}$ samples of hippocampal tissue or BDNF standards were added to the wells. Plates were then covered and incubated for 2 hours at room temperature. Aliquots $(100 \mu \mathrm{l})$ of antihuman BDNF pAb (diluted 1:500) were added to the wells, plates were incubated for 2 hours at room temperature and washed 5 times. Aliquots $(100 \mu \mathrm{l})$ of anti-immunoglobulin Y horseradish peroxidase (1:2000 dilution) were added to the wells and incubated for 1 hour at room temperature. Postincubation, plates were washed X5 and $100 \mu$ l of the enzyme substrate (room temperature TMB One Solution) was added to the wells, and incubated for approximately $15 \mathrm{~min}$ until a blue color formed in the wells. The reaction was stopped by the addition of $100 \mu \mathrm{l}$ of $1 \mathrm{M}$ hydrochloric acid to the wells. Plates were read at ${ }_{450} \mathrm{~nm}$ in a 96 -well plate reader, and BDNF concentrations were estimated for the standard curve.
Western Blot Analysis of ERK Expression. Frozen, dissected brain samples were quick thawed on ice, rinsed in $\mathrm{Krebs} / \mathrm{CaCl}_{2}$ solution and then homogenized on ice in a $10 \mathrm{mM}$ Tris- $\mathrm{HCl}, 50 \mathrm{mM}$ $\mathrm{NaCl}, 1 \% \mathrm{vol} / \mathrm{vol}$ Triton X-100 buffer containing a cocktail of both protease inhibitors (Roche) and phosphatase inhibitors (Sigma, Irl). Lysates were then sonicated on ice for 2 minutes and centrifuged at $12,000 \times \mathrm{g}$ for 5 minutes at $4{ }^{\circ} \mathrm{C}$ and the supernatants were assayed for protein concentration (Bradford, 1976). Equal amounts of protein $(15 \mu \mathrm{g})$ from each animal were resolved on $12 \%$ SDSpolyacrylamide gels and transferred to nitrocellulose membrane by electroblotting. Membranes were blocked for $2 \mathrm{hrs}$. with $5 \%$ bovine serum albumin (BSA) in $50 \mathrm{mM}$ Tris- $\mathrm{HCl}, 150 \mathrm{mM} \mathrm{NaCl}, 0.1 \%$ vol/vol Tween 20, pH 7.4 (TBST), then incubated overnight at $4{ }^{\circ} \mathrm{C}$ with primary antibodies against either phospho-ERK or total ERK (Cell Signaling Technology, Inc.) diluted 1:1000 in 2\% BSA/TBST. Membranes were stringently washed with TBST prior to $1 \mathrm{hr}$ incubation with a secondary horseradish peroxidase-conjugated anti-rabbit immunoglobulin G (IgG) antibody (Amersham Biosciences) diluted 1:3000 in $2 \%$ BSA/TBST. After stringent washing in TBST, blots were detected by enhanced chemiluminescence and exposed to film. Quantification was done using Quantity One software (version 4.41, Bio-Rad Laboratories, Inc.).

\section{Results}

Protein synthesis inhibition impairs long-term spatial memory retention and disrupts cue-platform relations in nonrotated animals. A $2 \times 5$ way mixed factorial ANOVA revealed that all animals acquired the maze, with escape latencies decreasing significantly from Day 1 to Day 5 of training, $F(4,88)=44.249, p<$ .001 ; Figure 3. As in Experiment 1 we found neither a significant group effect, $F(1,22)=0.350, p>.05$, nor a day $\times$ group interaction effect, $F(4,88)=1.841, p>.05$.

Seven days postacquisition, we subdivided both saline and CHX-treated groups into control and cue-rotated conditions. As an initial examination of the animals' retention of the maze we calculated the mean percentage time spent by the saline group in each of the four quadrants in the maze (see Figure $4 \mathrm{a}$, inset). If animals had successfully retained the maze, then CT should search predominantly in the NE quadrant, while CR should follow the

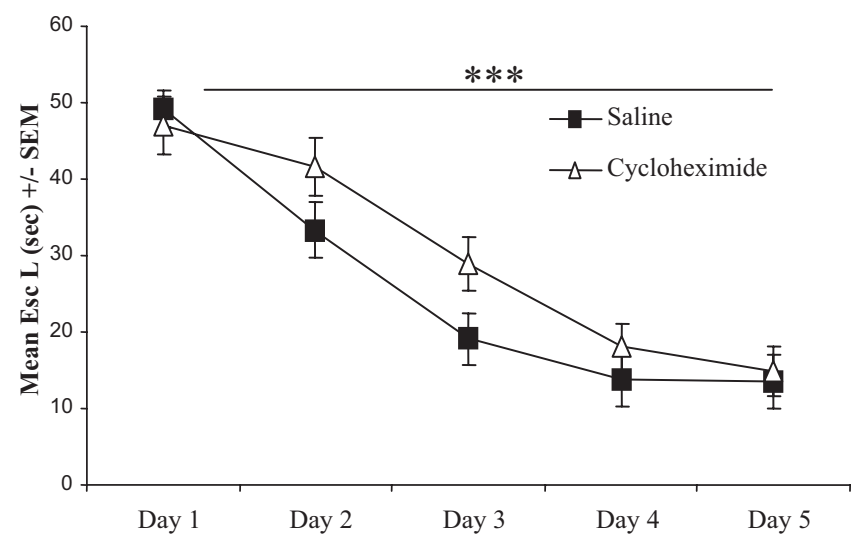

Figure 3. Mean Escape Latencies demonstrated by animals in cycloheximide (CHX) and animals in Group 1 (SAL) over the 5-day training phase (Experiment 2). 


\section{Saline}

a
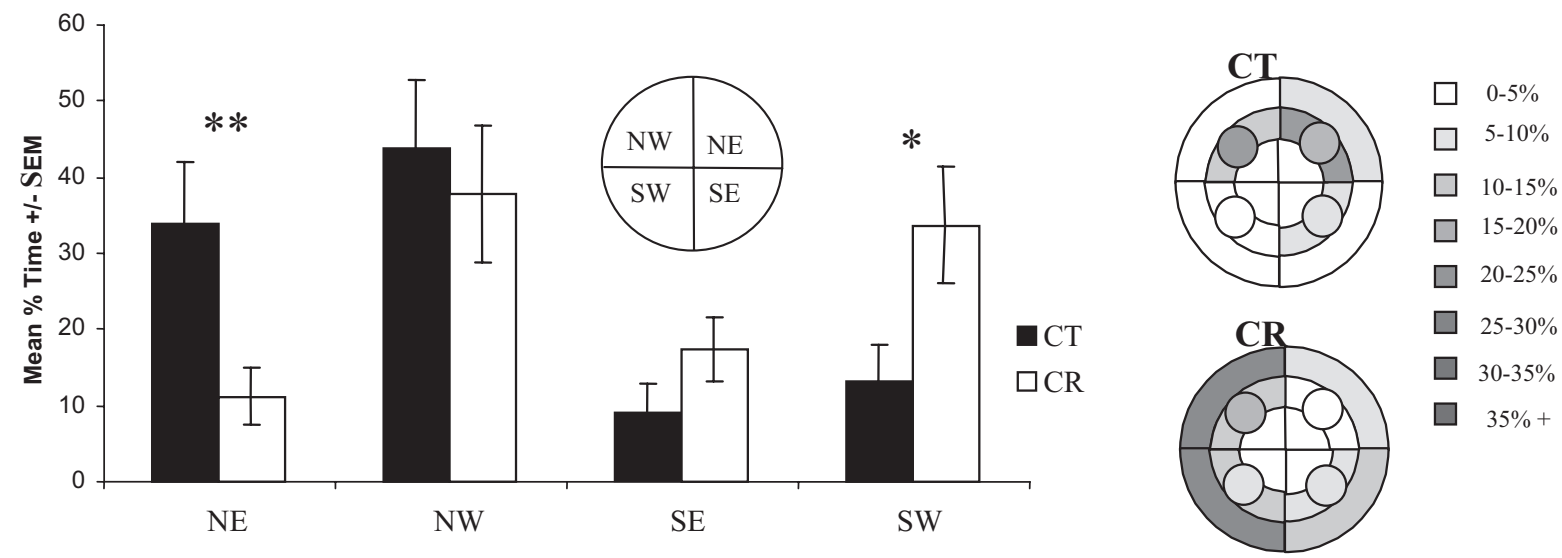

\section{Cycloheximide}
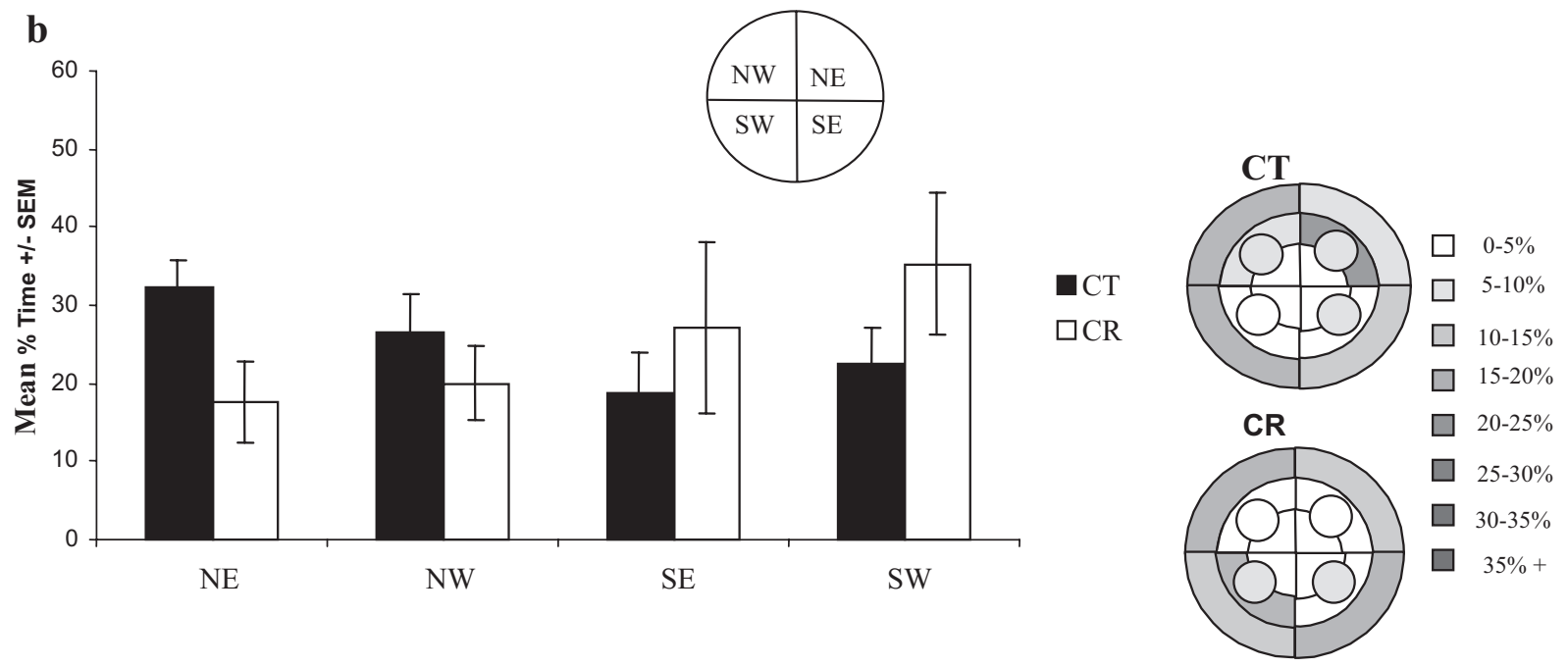

c

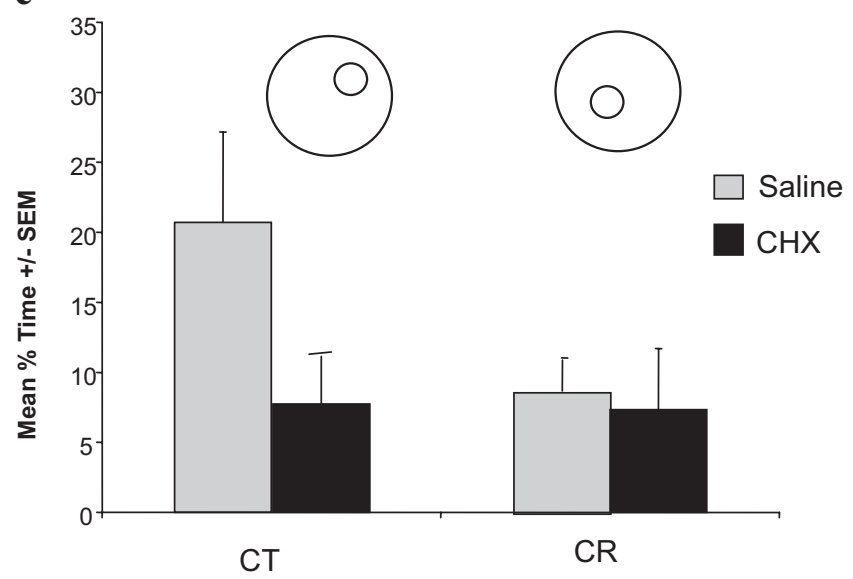

Figure 4. Mean percentage time (of the initial 30 seconds) spent by saline a and cycloheximide (CHX) b groups in each of the four quadrants. For the saline group, the animals in the (control) CT condition spent significantly more time in the northeast (NE) quadrant compared to the cue-rotated (CR) condition spent, while animals in the CR condition spent significantly more time in the southwest (SW) quadrant compared to the CT animals. The right panel of both a and b graphically represents the mean percentage time spent by both groups in various regions of the pool with the darker shading representing the more time in that region. It should be noted that saline animals in the CR condition spend a lot of time at the side of the SW quadrant something that we have noted before (McGauran et al., 2005). C, Mean percentage time (of the initial 30 seconds) spent by saline and CHX groups in their cue-dependent platform area (CT in NE and CR in SW). 
rotated cues, searching in the SW quadrant. A $2 \times 4$ repeatedmeasures ANOVA was performed on the swimming behavior of the SAL group, and revealed a significant main effect for quadrant, $F(3,11)=4.805, p<.05$. An interaction effect was also found, $F(3,11)=3.556, p<.05$. A series of independent $t$ tests were conducted in order to determine where this interaction effect took place. These analyses revealed that the CT group showed a significant preference for the NE quadrant compared to the CR group, $t(13)=2.649, p<.01$, and the CR group demonstrating a significant preference for the SW quadrant, $t(13)=-2.173, p<$ .05 , compared to the CT group (see Figure 4a). No other differences were noted. In addition, Figure $4 \mathrm{a}$ also demonstrates the mean percentage time spent by CT (top right panel) and CR animals (bottom right panel) in various subregions of the pool to give an overall representation of where each group spent swimming. In addition, a further $2 \times 4$ way ANOVA was also conducted on the percentage time spent in each quadrant by animals in the CHX condition (Figure 4b). This analysis revealed that animals in either CT or CR condition did not demonstrate retention of the maze, showing no significant preference for any quadrant in particular, $F(3,7)=1.016, p>.05$; Figure $4 \mathrm{~b}$. Figure $4 \mathrm{~b}$ also demonstrates the mean percentage time spent by CT (top right panel) and CR animals (bottom right panel) in various subregions of the pool again to give an overall representation of where each group spent swimming.

We then examined the amount of time spent by each group of animals in their cue-related platform area (i.e., CT group (saline and $\mathrm{CHX}$ ) in the NE area, and $\mathrm{CR}$ group (saline and $\mathrm{CHX}$ ) in the SW area; Figure $4 \mathrm{c}$ inset). A $2 \times 4$ way ANOVA revealed a significant effect for treatment $[F(1,20)=6.660, p<.05]$. In addition, there was a significant difference found between cue conditions (CT and CR), $F(1,20)=5.516, p<.05$, and an interaction between cue condition and treatment, $F(1,20)=5.061$, $p<.05$; Figure $4 c$. Post hoc analyses revealed that animals treated with $\mathrm{CHX}$ appeared to be impaired in their retention, and did not search in the precise area location (CT in NE area and CR in SW area). Rotated animals (CR) in saline group also did not search in the precise NE area. These animals tended to search at the side of the SW quadrant (as opposed to the exact area within the quadrant, also see Figure 4a right bottom panel). We have found this previously on a number of occasions and have suggested that this thigmotactic-like preference for the side of the SW quadrant may be as a result of animals having to deal with conflicting vestibular information compared to that given by the distal visual cues (see McGauran et al., 2005).

Protein synthesis inhibition resulted in a down-regulation of Both BDNF and ERK expression in the hippocampus. To determine the role of protein synthesis in the formation of allocentric representations, hippocampal BDNF expression in the CT group was compared to that of the CR group in both the SAL and CHX conditions (Figure 5a). A two-way between Groups ANOVA demonstrated a significant effect for treatment,. $F(1,12)=5.653$, $p<.05$. No significant effect was found between CR and CT groups, $F(1,12)=0.325, p>.05$, and we also did not find a treatment $\times$ group interaction effect, $F(1,12)=2.261, p>.05$.

Phospho-ERK (pERK) was measured as a percentage of total ERK (tERK) in order to calculate differences in hippocampal ERK expression between the various groups. Western blot analysis demonstrated a lower hippocampal pERK expression in the CHX group compared to the SAL group (See Figure $5 \mathrm{~b}$, inset). A $2 \times$ $2 \times 2$ (ERK type, ERK1 vs. ERK2; cue-group, CT vs. CR; treatment type, Saline vs. CHX) was conducted. No significant effect was found for ERK type, $F(1,22)=1.040, p>.05$ ) or cue-group, $F(1,22)=3.622, p>.05$ but there was a significant main effect for treatment (i.e., SAL or CHX; $F(1,22)=4.510$, $p<.05)$. In addition, there was no treatment $\times \mathrm{ERK}, F(1,22)=$ $0.384, p>.05$, treatment $\times$ cue type, $F(1,22)=0.011, p>.05$, or ERK $\times$ cue type interaction effects, $F(1,22)=1.134, p>.05$. Multiple $t$ tests were performed to investigate the main treatment effect, and these revealed that for both ERK1 and ERK2, CT animals which received CHX expressed less hippocampal ERK than their saline controls, ERK1: $t(6)=3.08, p<.05$; ERK2: $t(6)=3.10, p<.05$. However, similar to our findings on hippocampal BDNF expression, no treatment effect was found in CR groups, ERK1: $t(6)=0.26, p>.05$; ERK2: $t(6)=0.07, p>.05$.

\section{Discussion}

From our first experiment we demonstrate that animals in all groups acquired the watermaze task successfully, with no major differences in general escape latencies. This supports previous reports in the literature that have suggested that protein synthesis is necessary for long, but not short-term memory formation (Davis \& Squire, 1984; McGaugh, 1966; McGaugh, 2000). As many researchers would consider a 24-hr interval as long-term memory, we examined the first trial of each day subsequent to injection in terms of escape latency and percentage time spent swimming by all treated groups in the NE quadrant (where the platform was located), to determine whether CHX had an effect on performance. We found no significant difference between the groups in terms of escape latencies but found a small significant difference between the saline group and $\mathrm{CHX} 2$ group in terms of the time spent searching for the platform in the NE quadrant. These findings would suggest that a 24-hr retention of the platform location was subject to a minimal interference by the higher dose of $\mathrm{CHX}$, which is in contrast with our findings for retention tested 7 days postacquisition, which produced much larger deficits.

There is a large body of evidence supporting the need for protein synthesis in both the consolidation of new memories and the reconsolidation of existing ones (Davis \& Squire, 1984; Dudai \& Eisenburg, 2004; Sara, 2000). We administered the protein synthesis inhibitor $\mathrm{CHX}$ posttraining in order to inhibit consolidationdependent protein synthesis and block retention in the watermaze task. The findings indicate a dose-dependent effect where, at a high dose, $\mathrm{CHX}$ blocks retention of the task. Animals that received $1.0 \mathrm{mg} / \mathrm{kg}$ of cycloheximide (CHX2) demonstrated a profound impairment in watermaze retention when compared to both saline controls and animals that received a lower dose of $0.5 \mathrm{mg} / \mathrm{kg}$ cycloheximide (CHX1). CHX2 animals demonstrated no preference for the platform quadrant whatsoever when compared to the three other equivalent areas, and spent significantly less time swimming in this quadrant than saline controls. The findings of this study supported our expectations from the literature, with the lower dose of CHX not causing sufficient protein synthesis inhibition to impair memory, while the higher dose did. It is known that large doses of CHX produce a number of "nonspecific" adverse effects (Davis \& Squire, 1984) and at high doses it can be toxic to the animals. Hirate et al. (1997) for example, reported 2.8 
$\mathbf{a}$
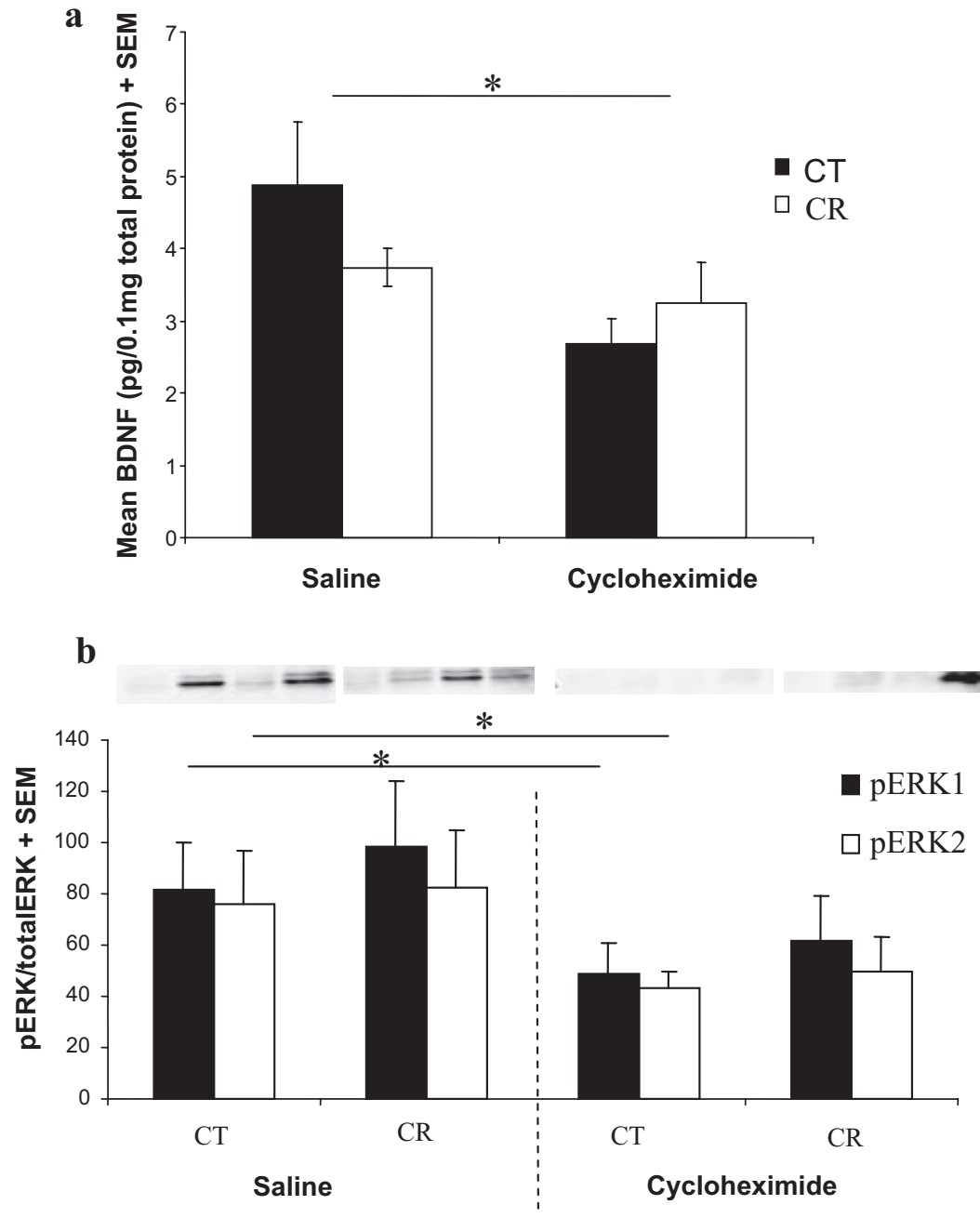

Figure 5. Hippocampal brain-derived neurotrophic factor (BDNF) and regulated kinase (ERK) expression in Experiment 2: A, BDNF expression in the hippocampus of animals in Group 1 (SAL) and cycloheximide (CHX) groups. CHX had significantly lower hippocampal expression than SAL; this difference was seen between the control (CT) but not the cue-rotated (CR) groups. B, ERK expression in the hippocampus of animals treated with either saline (SAL) or cycloheximide (CHX), in relation to their cue condition (CT and CR). These mirror behavioral and BDNF results with CHX showing less ERK expression than SAL in the CT condition, but not in the $\mathrm{CR}$ condition.

$\mathrm{mg} / \mathrm{kg}$ as the highest possible nonlethal dose for sc administration, while other researchers have used as little as $0.6 \mathrm{mg} / \mathrm{kg}$ (Luft et al., 2004). While we have found previously in our lab that a high dose of CHX leads to death in some animals. However, similar to other studies (Mierzejewski et al., 2006), we found that CHX administration did not produce any major locomotor or motivational deficits in the experiments reported earlier.

In Experiment 2, we examined the influence of protein synthesis inhibition on animals' ability to specifically retain cue-platform associations and how $\mathrm{CHX}$ may affect subsequent expression of BDNF and ERK in the hippocampus. As in Experiment 1, we confirmed that protein synthesis inhibition does not have an effect on the acquisition of the task. However, animals were unable to retain the location of the platform seven days postacquisition. Unfortunately as both saline- and CHX-treated animals were equally impaired following cue-rotation (certainly in terms of precise searching), we cannot draw any conclusions on the specific type of spatial processing that may be impaired as we had hoped in our introduction.

Here we also report a relationship between protein synthesis and hippocampal BDNF and ERK expression, where BDNF and ERK expression was higher in the saline group compared to the $\mathrm{CHX}$ group. Although both BDNF (Gooney \& Lynch, 2001; Han \& Holtzmann, 2000) and ERK (Adams \& Sweatt, 2002; Chandler, Sutton, Dorairaj, \& Norwood, 2001) have been found on numerous occasions to be independently important for protein synthesis, our findings may suggest a relationship or at least a parallelism between both BDNF and ERK during learning and memory processes, with both being mediated by the MAP-K pathway during protein synthesis. 
Whether or not this relationship between BDNF, ERK, and protein synthesis is as a result of direct interactions between these factors is unclear, but they certainly seem to play some important role in memory mechanisms. The process by which $\mathrm{CHX}$ itself specifically affected retention in our study needs yet to be fully investigated, but we demonstrate that, possibly via a number of intermediary molecular and cellular mechanisms, protein synthesis inhibition by CHX resulted in impaired retention seven days later. The main body of research has established that BDNF acts through the MAP-K pathway during protein synthesis, directly affecting the onset of protein synthesis (e.g., Finkbeiner, Tavozoie, Maloratsky, Jacobs, \& Greenberg, 1997; Krug, Lossner, \& Ott, 1984). Yet, there has been relatively little research into the possibility of this relationship running in the opposite direction, that is, does protein synthesis or the MAP-K pathway influence BDNF expression? The possibility of such a relationship has, however, been touched on in a small number of studies investigating the role of BDNF and protein synthesis in learning and memory. MAP-K has been demonstrated as one of the major signaling molecules to influence neurotrophic signaling (Kaplan \& Miller, 2000; Segal \& Greenberg, 1996). Inhibition of the MAP-K pathway has been shown to impair the effect of BDNF at the synapse (Gottschalk et al., 1999). Indeed, localized synaptic potentiation by BDNF requires local protein synthesis in the axon, with prolonged exposure to anisomycin inhibiting BDNF-induced synaptic signaling (Zhang \& Poo, 2002). In addition, BDNF-induced LTP in rat hippocampal slices is dependent on local protein synthesis (Kang \& Schuman, 1996). In a recent study, local infusion of anisomycin into the dorsal hippocampus blocked late-phase BDNF activity during an inhibitory avoidance task (Bekinschtein et al., 2007). Interesting to note, Gottschalk and colleagues (1999) found that, while BDNF activity was dependent on MAP-K activity (along with other signaling pathways), infusion of anisomycin or CHX did not affect BDNF activity itself. They suggest BDNF-modulation of high-frequency transmission to be independent of protein synthesis, but dependent on the MAP-K pathway. In relation to the current study, these findings may be interpreted as suggesting that the inhibition of protein synthesis may have affected ERK activity, through the MAP-K pathway; this inhibition of MAP-K could therefore have down-regulated BDNF activity and hence retention for the spatial task.

The process through which BDNF and the MAP-K cascade influence protein synthesis may hold an interesting key to understanding learning and memory processes in general. Previously thought to play only a role in neuron regeneration and survival, the activity of BDNF in synaptic plasticity has, since its discovery, received much research interest, and is fast becoming recognized as one of the main molecular players in learning and memory (see Yamada et al., 2002). The current study succeeds in confirming this important role played by BDNF, perhaps through its interaction with equally important molecules and processes, MAP-K, ERK and protein synthesis. This work offers a possible path whereby molecular and cellular processes may be investigated in a new light, to reap further knowledge on the underlying biochemical bases of learning and memory.

\section{References}

Adams, J. P., \& Sweatt, J. D. (2002). Molecular psychology: Roles for the ERK MAP kinase cascade in memory. Annual review of pharmacology and toxicology, 42, 135-163.
Agnihotri, N. T., Hawkins, R. D., Kandel, E. R., \& Kentros, C. (2004). The long-term stability of new hippocampal place fields requires new protein synthesis. Proceedings of the National Academy of Sciences of the United States of America, 101, 3656-3661.

Atkins, C. M., Selcher, J. C., Petraitis, J. J., Trzaskos, J. M., \& Sweatt, J. D. (1989). The MAPK cascade is required for mammalian associative learning. Nature Neuroscience, 1, 602-609.

Bao, S., Chen, L., Qiao, X., Knusel, B., \& Thompson, R. F. (1998). Impaired eye-blink conditioning in waggler, a mutant mouse with cerebellar BDNF deficiency. Learning \& Memory, 5, 355-364.

Barnett, S. A. (1963). The rat: A study in behavior. Chicago: Aldine.

Bear, M. F., Connors, B. W., \& Paradiso MA. (1995). Neuroscience exploring the brain: Chemical control of brain and behavior (pp. $402-$ 432). Baltimore: Williams \& Wilkins.

Bekinschtein, P., Cammarota, M., Müller-Igaz, L., Bevilaqua, L. R. M., Izquierdo, I., \& Medina, J. H. (2007). Persistence of long-term memory storage requires a late protein-synthesis and BDNF-dependent phase in the hippocampus. Neuron, 53, 261-277.

Blum, S., Moore, A. N., Adams, F., \& Dash, P. K. (1999). A mitogenactivated protein kinase cascade in the CA1/CA2 subfield of the dorsal hippocampus is essential for long-term spatial memory. Journal of Neuroscience, 19, 3535-3544.

Bourtchouladze, R., Abel, T., Berman, N., Gordon, R., Lapidus, K., \& Kandel, E. R. (1998). Different training procedures recruit either one or two critical periods for contextual memory consolidation, each of which requires protein synthesis and PKA. Learning \& Memory, 51, 365-374.

Bradford, M. M. (1976). A rapid and sensitive method for the quantitation of microgram quantities of protein utilizing the principle of protein-dye binding. Analytical Biochemistry, 72, 248-54.

Chandler, L. J., Sutton, G., Dorairaj, N. R., \& Norwood, D. (2001). $\mathrm{N}$-methyl D-aspartate receptor-mediated bidirectional control of extracellular signal-regulated kinase activity in cortical neuronal cultures. Journal of Biological Chemistry, 276, 2627-2636.

Commins, S., Cunningham, L., Harvey, D., \& Walsh, D. (2003). Massed but not spaced training impairs spatial memory. Behavioural Brain Research, 139, 215-223.

D'Hooge, R., \& De Deyn, P. P. (2001). Applications of the Morris water maze in the study of learning and memory. Brain Research Reviews, 36, 60-90.

Dash, P. K., Hebert, A. E., \& Runyan, J. D. A unified theory for systems and cellular memory consolidation. Brain Research Reviews, 45, 30-37.

Davies, A. M. (1994). The role of neurotrophins in the developing nervous system. Journal of Neurobiology, 25, 1334-1348.

Davis, H. P., \& Squire, L. R. (1984). Protein synthesis and memory: A review. Psychological Bulletin, 96, 513-559.

Debiec, J., LeDoux, J. E., \& Nader, K. (2002). Cellular and systems reconsolidation in the hippocampus. Neuron, 36, 527-538.

Derkinderen, P., Enslen, H., \& Girault, J.-A. (1999). The ERK/MAPKinases cascade in the nervous system. NeuroReport, 10, 24-34.

Dudai, Y., \& Eisenburg, M. (2004). Rites of passage of the engram: Reconsolidation and the lingering consolidation hypothesis. Neuron, 44, 93-100.

English, J. D., \& Sweatt, J. D. (1997). A requirement for the mitogenactivated protein kinase cascade in hippocampal long term potentiation. Journal of Biological Chemistry, 272, 19103-19106.

Finkbeiner, S., Tavozoie, S. F., Maloratsky, A., Jacobs, K. M., \& Greenberg, M. E. (1997). CREB: A major mediator of neuronal neurotrophin responses. Neuron, 19, 1031-1047.

Fischer, A., Sananbenesi, F., Schrick, C., Spiess, J., \& Radulovic, J. (2004). Distinct roles of hippocampal de novo protein synthesis and actin rearrangement in extinction of contextual fear. Journal of Neuroscience, 25, 1962-1966.

Gooney, M., \& Lynch, M. A. (2001). Long-term potentiation in the dentate gyrus of the rat hippocampus is accompanied by brain-derived neuro- 
trophic factor-induced activation of TrkB. Journal of Neurochemistry, 77, $1198-1207$.

Gottschalk, W. A., Jiang, H., Tartaglia, N., Feng, L., Figurov, A., \& Lu, B. (1999). Signalling mechanisms mediating BDNF modulation of synaptic plasticity in the hippocampus. Learning \& Memory, 6, 243-256.

Grecksch, G., \& Matthies, H. (1980). Two sensitive periods for the amnesic effect of anisomycin. Pharmacology Biochemistry and Behavior, $12,663-665$.

Hall, J., Thomas, K. L., \& Everitt, B. J. (2000). Rapid and selective induction of BDNF expression in the hippocampus during contextual learning. Nature Neuroscience, 3, 533-535.

Han, B. H., \& Holtzman, D. M. (2000). BDNF protects the neonatal brain from hypoxic-ischemic injury in vivo, via the ERK pathway. Journal of Neuroscience, 20, 5775-5781.

Hirate, K., Hirano, M., Nakajima, Y., Hiyama, A., Maeda, O., \& Asakura, W. (1997). No. 302, a newly synthesized [pGlu4,Cyt 6]AVP(4-9) analogue, prevents the disruption of avoidance behavior. Behavioural Brain Research, 83, 205-208.

Johnston, A. N. B., Clements, M. P., \& Rose, S. P. R. (1999). Role of brain-derived neurorophic factor and presynaptic proteins in passive avoidance learning in day-old domestic chicks. Neuroscience, 88, 1033 1042.

Kang, H., \& Schuman, E. M. (1996). A requirement for local protein synthesis in neurotrophin-induced hippocampal synaptic plasticity. Science, 273, 1402-1406.

Kaplan, D. R., \& Miller, F. D. (2000). Neurotrophin signal transduction in the nervous system. Current Opinion in Neurobiology, 10, 381-391.

Kesslak, J. P., So, V., Choi, J., Cotman, C. W., \& Gomez-Pinilla, F. (1998). Learning up regulates brain-derived neurotrophic factor messenger ribonucleic acid: A mechanism to facilitate encoding and circuit maintenance? Behavioral Neuroscience, 112, 1012-1019.

Krug, M., Lossner, B., \& Ott, T. (1984). Anisomycin blocks the late phase of long-term potentiation in the dentate gyrus of freely moving rats. Brain Research Bulletin, 13, 39-42.

Lewin, G. R., \& Barde, Y. A. (1996). Physiology of the neurotrophins. Annual Review of Neuroscience, 19, 289-317.

Lu, M-C., Lin, Y-C., \& Peng, W-H. (2004). Effects of san-yu-tang on the cycloheximide-induced amnesia in rats. Journal of Chinese Medicine, 15, 87-97.

Luft, A. R., Buitrago, M. B., Kaelin-Lang, A. Dichgans, J., \& Schulz1, J. B. (2004). Protein Synthesis Inhibition Blocks Consolidation of an Acrobatic Motor Skill. Learning \& Memory, 11, 379-382.

Müller, G. E., \& Pilzecker, A. (1900). Experimentelle beiträge zur lehre vom gedächtnis. [Experimental contributions to the science of memory]. Zeitschrift und Physiologie der Sinnesorgane, S, 1, 1-288.

McGaugh, J. L. (1966). Time-dependent processes in memory storage. Science, 153, 1351-1358.

McGaugh, J. L. (1999). The perseveration-consolidation hypothesis: Mueller and Pilzecker 1900. Brain Research Bulletin, 50, 445-446.

McGaugh, J. L. (2000). Memory: A century of consolidation. Science, 287, $248-251$.
McGauran, A. T., Harvey, D., Cunningham, L., Craig, S., \& Commins, S. (2004). Retention of cue-based associations in the water maze is timedependent and sensitive to disruption by rotating the starting position. Behavioural Brain Research, 151, 255-266.

McGauran, A. T., O’Mara, S. M., \& Commins, S. (2005). Vestibular influence on watermaze retention: Transient whole body rotations improve the accuracy of the cue-based retention strategy. Behavioural Brain Research, 158, 183-187.

Meiri, N., \& Rosenblum, K. (1998). Lateral ventricle injection of the protein synthesis inhibitor anisomycin impairs long-term memory in a spatial memory task. Brain Research, 789, 48-55.

Mierzejewski, P., Siemiatkowski, M., Radwanska, K., Szyndler, J., Bienkowski, P., Stefanski, R., et al. (2006). Cycloheximide impairs acquisition but not extinction of cocaine self-administration. Neuropharmacology, 51, 367-73.

Morris, R. G. M. (1984). Development of a watermaze procedure for studying spatial learning in the rat. Journal of Neuroscience Methods, $11,47-60$.

Quevedo, J., Vianna, M. R., Roesler, R., de-Paris, F., Izquierdo, I., \& Rose, S. P. (1999). Two time windows of anisomycin-induced amnesia for inhibitory avoidance training in rats: Protection from amnesia by pretraining but not pre-exposure to the task apparatus. Learning \& Memory, 6, 600-607.

Rossato, J. I., Bevilaqua, L. R. M., Myskiw, J. C., Medina, J. H., Izquierdo, I., \& Cammarota, M. (2007). On the role of hippocampal protein synthesis in the consolidation and reconsolidation of object recognition memory. Learning \& Memory, 14, 36-46.

Sara, S. J. (2000). Retrieval and reconsolidation: Toward a neurobiology of remembering. Learning \& Memory, 7, 73-84.

Segal, R. A., \& Greenberg, M. E. (1996). Intracellular signalling pathway activated by neurotrophic factors. Annual Review of Neuroscience, 19, 463-489.

Selcher, J. C. Atkins, C. M., Trzaskos, J. M., Paylor, R., \& Sweatt, J. D. (1999). A necessity for MAP Kinase activation in mammalian spatial learning. Learning \& Memory, 6, 107-137.

Tokuyama, W., Okuno, H., Hashimoto, T., Li, Y. X., \& Miyashita, Y. (2000). BDNF upregulation during declarative memory formation in monkey inferior temporal cortex. Nature Neuroscience, 3, 1134-1142.

Tyler, W. J., Alonso, M., Bramham, C. R., \& Pozzo-Miller, L. D. (2002). From Acquisition to Consolidation: On the Role of Brain-Derived Neurotrophic Factor Signaling in Hippocampal-Dependent Learning. Learning \& Memory, 9, 224-237.

Yamada, K., Mizuno, M., \& Nabeshima, T. (2002). Role for brain-derivedneurotrophic-factor in learning and memory. Life Sciences, 70, 735-744.

Zhang, X., \& Poo, M. (2002). Localized synaptic potentiation by BDNF requires local protein synthesis in the developing axon. Neuron, 36, 675-688.

Received October 16, 2007

Revision received January 8, 2008

Accepted February 20, 2008 